\title{
ANÁLISIS DE MATERIALES ARTÍSTICOS MEDIANTE ESPECTROSCOPIA INFRARROJA POR REFLECTANCIA TOTAL ATENUADA
}

ANALYSIS OF PICTORIAL MATERIALS BY ATTENUATED TOTAL REFLECTANCE FOURIER TRANSFORM INFRARED SPECTROSCOPY

ANÁLISE DE MATERIAIS ARTÍSTICOS UTILIZANDO ESPECTROSCOPIA DE ABSORÇÃO NO INFRAVERMELHO POR REFLECTÂNCIA TOTAL ATENUADA

Astrid Carolina Blanco Guerrero Marta Silvia Maier

Como citar este artigo:

BLANCO GUERRERO, Astrid Carolina; MAIER, Marta Silvia. Análisis de materiales artísticos mediante espectroscopia infrarroja por reflectancia total atenuada. In: Cadernos do Lepaarq, v. XV, n.30., p. 267-276, Jul-Dez. 2018. 


\title{
Análisis de materiales artísticos mediante espectroscopia infrarroja por reflectancia total atenuada
}

\author{
Astrid Carolina Blanco Guerrero* \\ Marta Silvia Maier*
}

Resumo: Este trabalho descreve a análise por espectroscopia de infravermelho usando a técnica de reflectância total atenuada de tintas a óleo e acrílicos comerciais contendo branco de titânio, azul da Prússia e verde de ftalocianina como pigmentos. As pinturas a óleo com azul da Prússia mostraram a presença de cargas como carbonato de cálcio e sulfato de bário. A análise de uma réplica de uma tinta acrílica com branco de titânio sobre a qual foi aplicada uma camada de verniz Paraloid B-67 produziu um espectro infravermelho correspondente ao verniz, não registrando bandas da camada de formulação com branco de titânio. Por outro lado, no óleo e acrílicos com verde de ftalocianina, ambos os aglutinantes foram claramente diferenciados e o pigmento foi identificado com base em alguns sinais característicos em áreas de fingerprint. Os resultados mostram a versatilidade da técnica vibracional no estudo de materiais artísticos.

\section{Palavras Chave:}

FTIR-ATR; Polímeros acrílicos; Pinturas a óleo.
Resumen: este trabajo describe el análisis por espectroscopia infrarroja utilizando la técnica de reflectancia total atenuada de pinturas al óleo y acrílicas comerciales conteniendo blanco de titanio, azul de Prusia y verde de ftalocianina como pigmentos. Las pinturas al óleo con azul de Prusia mostraron la presencia de cargas como carbonato de calcio y sulfato de bario. El análisis de una réplica de una pintura acrílica con blanco de titanio sobre la que se aplicó una capa de barniz Paraloid B-67 dio un espectro infrarrojo correspondiente al barniz, no registrándose bandas de la capa de la formulación con blanco de titanio. Por otra parte, en las pinturas al óleo $\mathrm{y}$ acrílicas con verde de ftalocianina se diferenciaron claramente ambos aglutinantes y se identificó el pigmento en base a algunas señales características en la zona de la huella digital. Los resultados muestran la versatilidad de la técnica vibracional en el estudio de materiales artísticos.

\section{Palabras claves:}

FTIR-ATR; Polímeros acrílicos; Pinturas al óleo.

\begin{abstract}
: this work describes the analysis by attenuated total reflectance infrared spectroscopy of oil and acrylic commercial paintings with titanium white, Prussian blue and green phthalocyanine as pigments. Oil paintings with Prussian blue showed the presence of calcium carbonate and barium sulfate as extenders. Analysis of a mock-up of an acrylic painting with titanium white with the application of Paraloid B-67 as varnish showed the infrared spectrum of the varnish and no bands of the titanium white painting. On the other hand, in the oil and acrylic paintings with green phthalocyanine both binders were clearly differentiated and the pigment was identified by some characteristic bands in the fingerprint region. Our results show the versatility of the vibrational technique in the study of pictorial materials.
\end{abstract}

\section{Keywords:}

FTIR-ATR; Acrylic polymers;

Oil paints.

\footnotetext{
* UMYMFOR-CONICET y Departamento de Química Orgánica, Facultad de Ciencias Exactas y Naturales, Universidad de Buenos Aires. Pabellón 2, Ciudad Universitaria, (C1428EGA), Ciudad Autónoma de Buenos Aires, Argentina.

E-mail: astridcbg@gmail.com.
} 


\section{INTRODUCCIÓN}

Las formulaciones comerciales de pinturas artísticas modernas utilizan principalmente aceites vegetales y resinas acrílicas como aglutinantes de pigmentos inorgánicos y orgánicos. Otros componentes, tales como carbonato de calcio $\left(\mathrm{CaCO}_{3}\right)$, talco $\left(\mathrm{Mg}_{3}\left(\mathrm{Si}_{4} \mathrm{O}_{10}\right)(\mathrm{OH})_{2}\right)$, barita $\left(\mathrm{BaSO}_{4}\right)$, caolinita $\left(\mathrm{Al}_{2} \mathrm{Si}_{2} \mathrm{H}_{4} \mathrm{O}_{9}\right)$, y diferentes formas de sílice se agregan para facilitar la extensión de la pintura, disminuir su costo y modificar su calidad. Los pigmentos inorgánicos comprenden óxidos de hierro, titanio y zinc, ferrocianuro férrico (azul de Prusia) y azul ultramar, un complejo sintético de óxidos de sodio, aluminio y silicio y sulfuro de sodio. Desde el siglo XX, la mayor parte de los pigmentos orgánicos utilizados en las formulaciones son compuestos sintéticos que se clasifican de acuerdo a su estructura química, tales como quinacridonas, ftalocianinas o compuestos azoicos, entre otros (LEARNER 2004).

La complejidad de estas formulaciones requiere de la aplicación de una combinación de técnicas analíticas para su caracterización en obras de arte. Entre éstas, la espectroscopia Raman permite identificar pigmentos, tanto inorgánicos como orgánicos, mientras que las técnicas de espectrometría de masa acopladas a cromatografía gaseosa, con o sin una etapa de pirólisis, han demostrado ser aplicables para la identificación de aceites vegetales y resinas acrílicas. Por otra parte, la espectroscopia infrarroja es una técnica vibracional adecuada para identificar tanto componentes inorgánicos como orgánicos. Desde hace varios años, la espectroscopia infrarroja por reflectancia total atenuada (FTIR-ATR) ha sido aplicada para el análisis de materiales en obras de arte (DERRICK et al. 1999) y actualmente es muy utilizada en estudios de degradación de formulaciones pictóricas modernas (PINTUS y SCHREINER 2011). Dependiendo de su composición, en muchos casos es factible identificar en un mismo análisis el pigmento, el tipo de aglutinante y los componentes agregados para extender la pintura.

Para una mejor interpretación de los resultados de la aplicación de la espectroscopia infrarroja para identificar y caracterizar los materiales artísticos, se requiere de una base de espectros infrarrojo de materiales de referencia (AGUAYO et al.2016). En nuestro laboratorio hemos iniciado recientemente el estudio de materiales en pinturas modernas y contemporáneas en el marco de un proyecto de investigación interdisciplinario que aborda la obra del artista argentino Antonio Berni (19051981) desde distintas perspectivas, entre ellas la caracterización de los materiales en obras destacadas del período 1950-1980. Este proyecto nos motivó para analizar por FTIR-ATR una serie de materiales que comprenden pigmentos inorgánicos y orgánicos, aceites vegetales, resinas acrílicas y formulaciones de pinturas artísticas comerciales con el objeto de conformar una biblioteca de espectros infrarrojo de referencia. En este trabajo presentamos los resultados del análisis por FTIR-ATR de formulaciones comerciales de pinturas al óleo y acrílicas conteniendo blanco de titanio (PW6, óxido de titanio), azul de Prusia (PB27, ferrocianuro férrico) y verde de ftalocianina (PG7) como pigmentos. El blanco de titanio presenta un alto poder cubriente, un costo accesible y, a diferencia del blanco de plomo, no es tóxico (GETTENS et al. 1993). Estas propiedades lo han posicionado como el pigmento blanco por excelencia, aunque a veces se lo mezcla con óxido de zinc. El azul de Prusia es un pigmento inorgánico sintético de gran poder de tinción y de un color azul profundo e intenso (BERRIE 1997), mientras que el verde de ftalocianina es un pigmento organometálico clorado conteniendo cobre y que posee excelentes propiedades en cuanto a su brillo, intensidad y permanencia (LOMAX 2005). 


\section{MATERIALES Y METODOS}

\section{Espectroscopia infrarroja}

Los espectros infrarrojo se registraron con un espectrofotómetro Thermo Scientific Nicolet iS50 FTIR con un accesorio de reflectancia total atenuada iS50 ATR con un cristal de diamante. Para cada muestra, se registraron 64 espectros en el rango de 4000-400 $\mathrm{cm}^{-1}$ con una resolución de $4 \mathrm{~cm}^{-1}$. El espectro de aire fue registrado como fondo.

\section{Selección de muestras}

En la Tabla 1 se indican las muestras de referencia (aceites vegetales, pigmentos y cargas) y las formulaciones comerciales analizadas por FTIR-ATR.

\section{Preparación de muestras}

Las formulaciones de pinturas al óleo y acrílicas se aplicaron sobre portaobjetos de vidrio mediante una espátula metálica mientras que el barniz acrílico se aplicó con pincel. Para la preparación de la muestra de barniz acrílico sobre la pintura acrílica con blanco de titanio, una vez seca la capa de la formulación con el pigmento, se aplicó una capa fina de barniz acrílico con pincel. Las réplicas se secaron hasta peso constante. Las pinturas acrílicas se secaron luego de 2-5 minutos. Las formulaciones al óleo con aceite de lino requirieron de 1 a 2 semanas mientras que aquellas conteniendo aceite de girasol requirieron aproximadamente 2 meses. Dado que no fue factible lograr un contacto efectivo entre la capa de pintura sobre el portaobjeto con el cristal de diamante del ATR, se separó una película de pintura (alrededor de 4 mg) de cada portaobjeto con la ayuda de una espátula y se registró el espectro infrarrojo de la superficie de la capa. Los aceites de lino y girasol de referencia y las muestras de pigmentos y cargas no requirieron una preparación previa para su análisis por FTIR-ATR.

\section{RESULTADOS Y DISCUSIÓN}

\section{Pintura acrílica con blanco de titanio como pigmento}

El medio acrílico (Acrílico Gel Medium) y la pintura acrílica con óxido de titanio, ambas de la marca Alba, no indicaban en sus envases el tipo de emulsión acrílica empleada por el fabricante. Los resultados del análisis por FTIR-ATR de ambas formulaciones se indican en la Tabla 2 y los espectros infrarrojo se muestran en la Figura 1. En ambos casos se observan bandas características de tensiones C-H $\left(2970-2870 \mathrm{~cm}^{-1}\right)$, tensión del grupo carbonilo a $1725 \mathrm{~cm}^{-1}$, dos bandas a 1449 y $1436 \mathrm{~cm}^{-1}$ correspondientes a la flexión antisimétrica del grupo metilo y la banda a $1386 \mathrm{~cm}^{-1}$ debida a la flexión simétrica del mismo grupo. Las dos bandas de tensión C-O a 1162 y $1143 \mathrm{~cm}^{-1}$ y la banda de torsión del grupo metileno alrededor de $842 \mathrm{~cm}^{-1}$ son características de los polímeros acrílicos. La comparación de los valores de las bandas de ambos espectros con datos de literatura (LEARNER 2004, ANGHELONE et al. 2017) indicaría la presencia de un copolímero de $n$-butilacrilato/metilmetacrilato (nBA/MMA). En general, la banda correspondiente al grupo carbonilo presenta valores similares para diferentes resinas acrílicas y por consiguiente no puede ser utilizada para su diferenciación. La confirmación de la composición de ambos polímeros acrílicos requiere un análisis por técnicas de espectrometría de masa (LEARNER 2004). En la formulación con dióxido de titanio se observa, además, la banda característica del pigmento a $483 \mathrm{~cm}^{-1} \mathrm{debida}$ a la vibración de tensión Ti-O (KAKUMA et al. 2015). La banda ancha entre 3000 y $3700 \mathrm{~cm}^{-1}$ se puede atribuir a la vibración de 
tensión del grupo $\mathrm{O}-\mathrm{H}$ de agua adsorbida sobre el pigmento. Ninguna de las bandas del pigmento interfiere con la asignación de las bandas del polímero acrílico (Figura 1).

El barniz acrílico de Winsor \& Newton (Oil Colour Varnishes) es un homopolímero de isobutilmetacrilato (conocido como Paraloid B-67), según se describe en el envase. En la Tabla 2 se indican las bandas principales en su espectro infrarrojo, destacándose dos bandas a 1389 y $1368 \mathrm{~cm}^{-1}$ características de la flexión simétrica C-H del grupo metilo en un isobutilmetacrilato junto con una mayor intensidad de la banda a $993 \mathrm{~cm}^{-1}$ (LEARNER 2004).

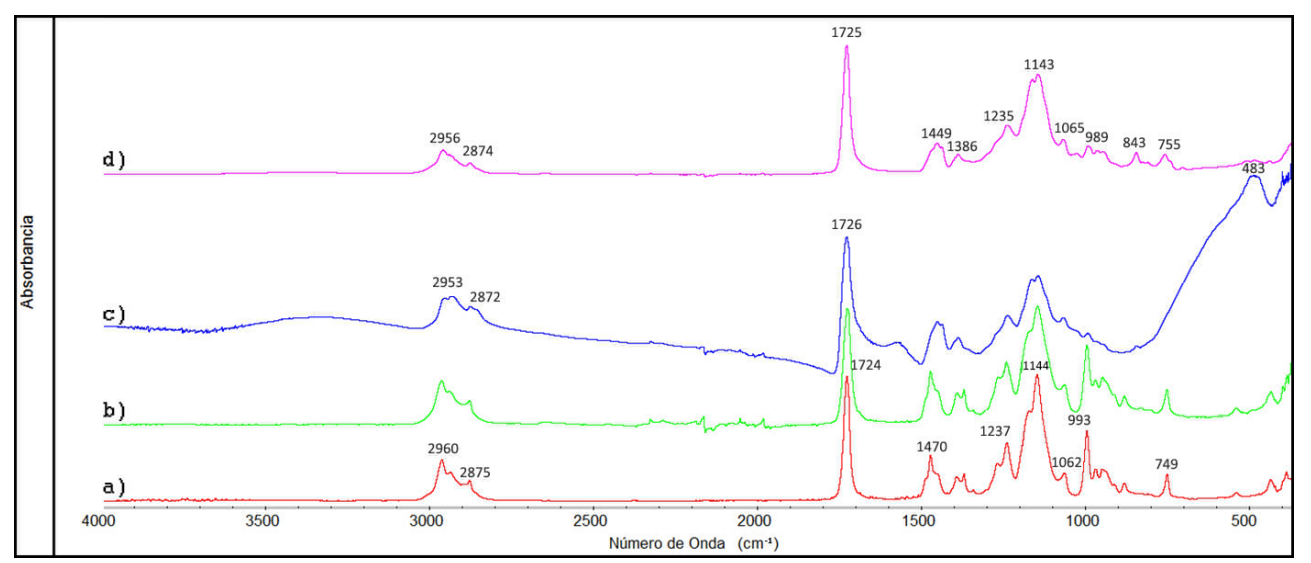

Figura 1: Espectros FTIR-ATR de las muestras de: a) Barniz W\&N, b) Barniz W\&N aplicado sobre la capa de pintura acrílica con blanco de titanio (ALBA), c) Pintura acrílica con blanco de titanio (ALBA) y d) Acrílico Gel Medium (ALBA).

En la Figura 1 se muestra el espectro infrarrojo de la réplica preparada con la pintura acrílica con blanco de titanio (Alba) sobre la cual se aplicó una capa de barniz acrílico (Paraloid B-67) y su comparación con el espectro infrarrojo de Paraloid B-67. Se observa claramente una coincidencia entre las bandas de ambos espectros infrarrojo y la ausencia de la banda característica del blanco de titanio a $483 \mathrm{~cm}^{-1}$ indicando que se registra únicamente el espectro del barniz Paraloid B-67 por encontrarse en la capa más externa de la réplica, una característica de la espectroscopia infrarroja por reflectancia total atenuada.

\section{Pinturas al óleo con azul de Prusia como pigmento}

Se analizaron por FTIR-ATR tres formulaciones de pintura al óleo conteniendo azul de Prusia (PB27) como pigmento de tres marcas diferentes. Las tres formulaciones contienen aceite de lino como vehículo, tal como se especifica en sus envases. Los tres espectros infrarrojo (Tabla 3) presentan las bandas principales del azul de Prusia alrededor de 2080 , 604 y $495 \mathrm{~cm}^{-1}$, además de una banda ancha entre 3200 y $3500 \mathrm{~cm}^{-1}$ y bandas débiles a 1610 y $980 \mathrm{~cm}^{-1}$ aproximadamente (LEARNER 2004). También se observan las bandas características del aceite de lino alrededor de 2920 y $2850 \mathrm{~cm}^{-1}$ (tensión C-H) y $1740 \mathrm{~cm}^{-1}$ (tensión del grupo carbonilo). Las mayores diferencias se observan en la zona de la huella digital de los tres espectros (Figura 2) debido a la presencia de componentes inorgánicos utilizados como cargas. Las formulaciones de Louvre Oil y Winton 33 presentan bandas características de carbonato de calcio $\left(\mathrm{CaCO}_{3}\right)$. En el espectro de Louvre Oil se observa una banda ancha e intensa a $1413 \mathrm{~cm}^{-1}$ característica del grupo carbonato y bandas agudas a 871 y $713 \mathrm{~cm}^{-1}$ que coinciden con las bandas características del carbonato de calcio (LEARNER 2004). La formulación de Winsor \& Newton (WINTON 33 ) presenta la banda de carbonato a $1413 \mathrm{~cm}^{-1}$ y dos bandas a 878 y $728 \mathrm{~cm}^{-1}$ que coinciden con las del espectro infrarrojo de la tiza comercial. Por otro lado, el espectro de la pintura de Utrecht Oil Color muestra bandas a 1177, 1069, 983,637 y 606 $\mathrm{cm}^{-1}$ coincidentes con la presencia de sulfato de bario (LEARNER 2004). Las bandas a 983 y $606 \mathrm{~cm}^{-1}$ se superponen a las 
correspondientes al azul de Prusia.

Tanto el carbonato de calcio como el sulfato de bario son sólidos inorgánicos inertes que se agregan como cargas a las formulaciones comerciales de pinturas al óleo para aumentar su volumen, modificar su textura y propiedades reológicas y reducir su costo (DOERNER 1998).

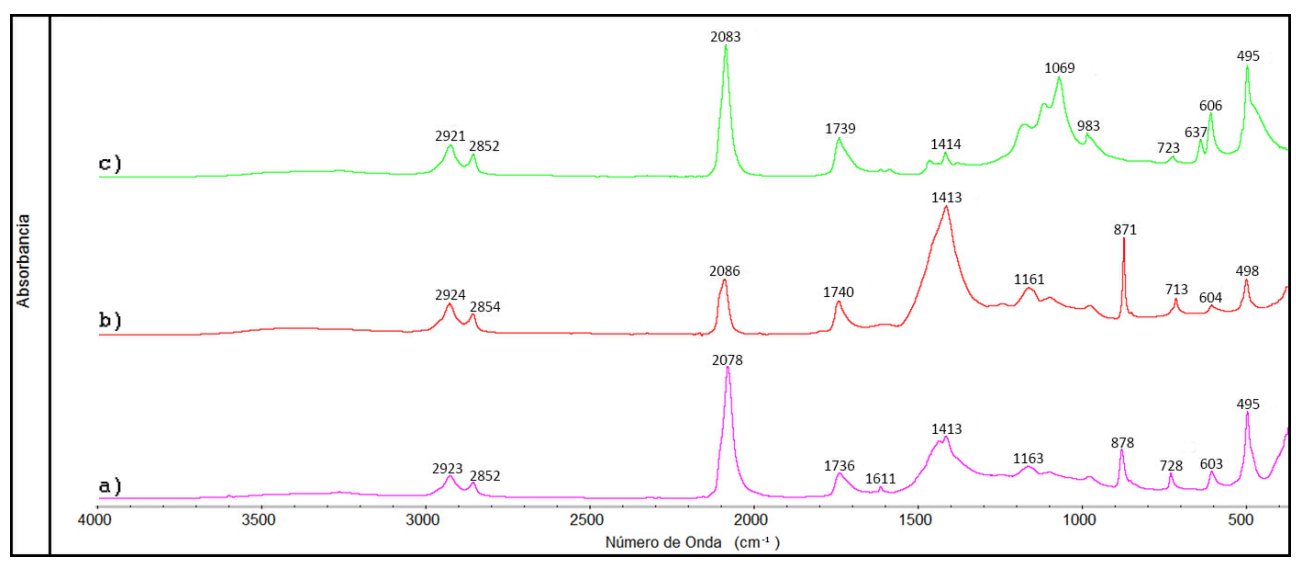

Figura 2: Espectros FTIR-ATR de las muestras de pintura al óleo con azul de Prusia (PB27): a) WINTON 33, W\&N, b) Louvre Oil y c) Utrecht Oil Color.

\section{Pinturas conteniendo verde de ftalocianina como pigmento}

En la Figura 3 se muestran los espectros infrarrojo de las formulaciones de pintura al óleo y acrílica conteniendo el pigmento sintético verde de ftalocianina (PG7). La formulación al óleo contiene aceite de girasol, tal como se especifica en el envase. Si bien el aceite de girasol es un aceite secante, su composición en ácidos grasos insaturados es diferente a la del aceite de lino al tener una menor proporción de ácidos grasos insaturados, lo cual aumenta su tiempo de secado (MILLS y WHITE 1994). Debido al alto poder de tinción de las ftalocianinas, se utilizan en pequeñas cantidades en las formulaciones de pintura. En consecuencia, su presencia se identifica en ambos espectros a partir de señales de poca intensidad a 1390, 1305, 949, 770 y $747 \mathrm{~cm}^{-1}$. Ambos espectros difieren en la posición de la banda del grupo carbonilo $\left(1727 \mathrm{~cm}^{-1}\right.$ para el acrílico y $1740 \mathrm{~cm}^{-1}$ para el óleo), lo cual permite diferenciarlos. En el espectro de la pintura acrílica, las bandas a $1237,1143,990$ y 843 $\mathrm{cm}^{-1}$ indicarían la presencia de un copolímero de $n$-butilacrilato/metilmetacrilato (nBA/MMA), al igual que en la formulación del medio acrílico de la misma marca (Fig. 1d).

En el espectro de la pintura al óleo conteniendo PG7, se observan las bandas características de la tensión C-H a 2924 y $2854 \mathrm{~cm}^{-1}$, antisimétrica y simétrica respectivamente, y la del carbonilo del aceite a $1740 \mathrm{~cm}^{-1}$. En la zona de la huella digital se observa una banda intensa a $1066 \mathrm{~cm}^{-1}$ junto con bandas a 986,637 y $609 \mathrm{~cm}^{-1}$ características de sulfato de bario. La presencia de una banda intensa a $441 \mathrm{~cm}^{-1}$ y el ensanchamiento de la banda alrededor de $1100 \mathrm{~cm}^{-1}$ indicaría el agregado de sílica como carga, además de sulfato de bario. 


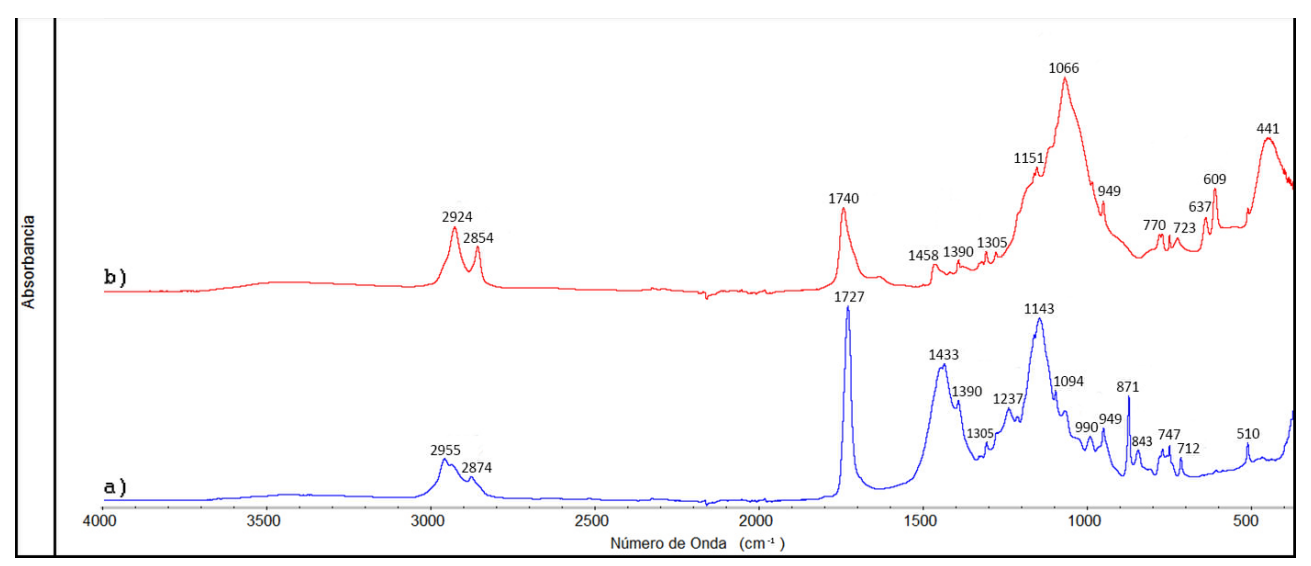

Figura 3: Espectros FTIR-ATR de las muestras conteniendo verde de ftalocianina (PG7): a) Acrílico Verde Ftalo 858 (ALBA) y b) Óleo Verde Ftalo 658 (ALBA).

\section{CONCLUSIONES}

Los análisis de las formulaciones pictóricas descriptas anteriormente muestran la versatilidad de la espectroscopia infrarroja para el análisis de componentes orgánicos e inorgánicos en un mismo análisis. La aplicación de la técnica de reflectancia total atenuada (ATR) no requiere de una preparación previa de las muestras y permite obtener espectros infrarrojo de calidad en unos pocos minutos.

Los resultados obtenidos demuestran que es posible identificar la presencia de un polímero acrílico o de un aceite vegetal en una formulación pictórica a partir de señales diagnósticas. Por otra parte, es común la presencia de componentes inorgánicos utilizados como cargas en las pinturas al óleo y estos pueden ser fácilmente identificables a partir de bandas características en la zona de la huella digital del espectro. Si bien en las pinturas acrílicas también se agregan aditivos, como por ejemplo surfactantes necesarios para mantener la estabilidad de la dispersión, es difícil identificarlos por FTIR y se requiere de la aplicación de técnicas analíticas más sensibles como espectrometría de masa o resonancia magnética nuclear (CICCOLA et al. 2017).

La espectroscopia infrarroja, en particular la técnica de ATR, brinda información de manera rápida y sencilla sobre la composición de una pintura artística y permite identificar el tipo de aglutinante orgánico, los pigmentos y otros componentes que forman parte de su formulación. A menudo esta técnica se aplica en combinación con la fluorescencia de rayos X (SHUGAR y MASS 2012) que brinda la composición elemental de una muestra y con la espectroscopia Raman, una técnica molecular muy versátil para la identificación de pigmentos y componentes inorgánicos utilizados como cargas en las formulaciones pictóricas (EDWARDS y CHALMERS 2005).

\section{Agradecimientos}

Las autoras agradecen a la Agencia Nacional de Promoción Científica y Tecnológica (ANPCyT) (Proyecto PICT2015-0440), al Consejo Nacional de Investigaciones Científicas y Técnicas (CONICET) (Proyecto PIP2014-2016, 11220130100288) y a la Universidad de Buenos Aires (Proyecto 20020130100008BA) por el apoyo financiero. A.C.B.G. agradece a CONICET por una beca doctoral. M.S.M. es miembro de la Carrera del Investigador Científico y Tecnológico de

\section{CONICET.}




\section{REFERENCIAS}

AGUAYO ALVARADO, Tomás; VARGAS GARCIA, Salvador; ESPINOSA IPINZA, Fernanda y GODOY TORRES, Valeria. Base de datos de espectros FT-IR de materiales usados en restauración: un aporte desde la ciencia a un público especializado. Conserva, 21, 145-151. 2016.

ANGHELONE, Marta; JEMBRIH-SIMBURGER, Dubravka y PINTUS, Valentina. Photostability and influence of phthalocyanine pigments on the photodegradation of acrylic paints under accelerated solar radiation. Polymer Degradation and Stability, 146, 13-23. 2017.

BERRIE, Barbara H. Prussian Blue. IN: WEST FITZHUGH, Elisabeth. Artists' Pigments. Volume 3 (pp. 191-217). Oxford: Oxford University Press. 1997.

EDWARDS, Howell G.M. y CHALMERS, John M. Raman spectroscopy in archaeology and art history. Royal Society of Chemistry, Cambridge, 2005.

PELEGRIN, Jacques. Taille. In: LEROI-GOURHAN, André. Dictionnaire de la Préhistoire (pp. 1019-1020). Paris: Press Universitaires de France. 1988.

CICCOLA, Alessandro; GUISO, Marcella; DOMENICI, Fabio; SCIUBBA, Fabio y BIANCO, Armandodoriano. Azopigments effect on UV degradation of contemporary art pictorial film: A FTIR-NMR combination study. Polymer Degradation and Stability, 140, 74-83. 2017.

DERRICK, Michele R.; STULIK, Dusan y LANDRY, James M. Infrared Spectroscopy in Conservation Science. The Getty Conservation Institute, Los Angeles, 1999.

DJAOUED, Yahia; BADILESCU, Simona, ASHRIT, Pandurang V., BERSANI, Danilo, LOTTICI, Pier P. y BRÜNING, Ralf. Low temperature sol-gel preparation of nanocrystalline $\mathrm{TiO}_{2}$ thin films. Journal of Sol-Gel Science and Technology, 24, 247-254. 2002.

DOERNER, Max. Los materiales de la pintura y su empleo en el arte. Reverté, Barcelona, 1998.

GETTENS, Rutherford J.; KÜHN, Hermann y CHASE, WilliamT. Lead White. IN: ROY, Ashok. Artists' Pigments. Volume 2 (pp. 67-81). Oxford: Oxford University Press. 1993.

LEARNER, Tom. Analysis of modern paints. The Getty Conservation Institute, Los Angeles, 2004.

LOMAX, Suzanne Q. Phthalocyanine and quinacridone pigments: their history, properties and use. Reviews in Conservation, 6, 19-30. 2005.

MILLS, John S. y WHITE, Raymond. The Organic Chemistry of Museum Objects. Butterworth-Heinemann, Oxford. 1994.

PINTUS, Valentina y SCHREINER, Manfred. Characterization and identification of acrylic binding media: influence of UV light on the aging process. Analytical and Bioanalytical Chemistry, 399, 2961-2976. 2011.

SHUGAR, Aaron N. y MASS Jennifer L. Handheld XRF for Art and Archaeology. Leuven University Press, Leuven. 2012. 


\section{ANEXOS}

Tabla 1: Lista de muestras estudiadas y sus características químicas y técnicas.

\begin{tabular}{|c|c|c|c|c|}
\hline \multicolumn{2}{|c|}{ Materiales de referencia } & \multicolumn{3}{|c|}{ Formulaciones comerciales } \\
\hline Producto & Proveedor & Producto & Proveedor & $\begin{array}{c}\text { Composición reportada por } \\
\text { el fabricante }\end{array}$ \\
\hline Sulfato de bario & $\begin{array}{l}\text { Mallinckrodt } \\
\text { (Analytical Reagent) }\end{array}$ & $\begin{array}{l}\text { Acrílico Blanco de Titanio } \\
810\end{array}$ & Alba & $\begin{array}{l}\begin{array}{l}\text { Emulsión } \\
\text { (vehículo) }\end{array} \\
\begin{array}{l}\text { Dióxido de } \\
\text { (pigmento) }\end{array}\end{array}$ \\
\hline Aceite de girasol & Cañuelas & $\begin{array}{lll}\text { Acrílico } & \text { Gel } & \text { Medium } \\
8204-996 & & \\
\end{array}$ & Alba & Emulsión acrílica \\
\hline $\begin{array}{l}\text { Carbonato de calcio } \\
\text { (Tiza) }\end{array}$ & $\begin{array}{l}\text { Balvanera Industrial } \\
\text { Artística }\end{array}$ & Oil Colour Varnishes & Winsor \& Newton & Metacrilato de isobutilo \\
\hline $\begin{array}{l}\text { Pigmento puro Titanium } \\
\text { White }\end{array}$ & $\begin{array}{l}\text { L. CORNELISSEN \& } \\
\text { SON }\end{array}$ & Óleo Verde Ftalo 658 & Alba & $\begin{array}{l}\text { Aceite purificado de girasol } \\
\text { (vehículo) } \\
\text { Ftalocianina cúprica clorada } \\
\text { PG7 (pigmento) }\end{array}$ \\
\hline $\begin{array}{l}\text { Pigmento puro Phthalo } \\
\text { (Mona) Green }\end{array}$ & $\begin{array}{l}\text { L. CORNELISSEN \& } \\
\text { SON }\end{array}$ & Acrílico Verde Ftalo 858 & Alba & $\begin{array}{l}\text { Emulsión acrílica pura } \\
\text { (vehículo) } \\
\text { Ftalocianina cúprica clorada } \\
\text { PG7 (pigmento) }\end{array}$ \\
\hline Aceite de linaza refinado & Winsor \& Newton & $\begin{array}{l}\text { Oil Color } \text { Permanent } \\
\text { Artists'Color }\end{array}$ & Utrecht & $\begin{array}{l}\text { Alkali Refined Linseed Oil } \\
\text { (vehículo) } \\
\text { Prussian Blue PB27 (pigmento) }\end{array}$ \\
\hline $\begin{array}{l}\text { Pigmento puro } \\
\text { Azul de Prusia }\end{array}$ & Winsor \& Newton & $\begin{array}{l}\text { OIL COLOUR WINTON } \\
33\end{array}$ & Winsor \& Newton & $\begin{array}{l}\text { Aceite de linaza (vehículo) } \\
\text { Ferricianuro álcali ferro PB27 }\end{array}$ \\
\hline- & - & Oil Colour & Louvre & $\begin{array}{lr}\begin{array}{l}\text { Huile de Lin } \\
\text { (vehículo) }\end{array} & \text { Decoloree } \\
\text { Ferri-ferrocyanide } & \text { PB27 } \\
\text { (pigmento) } & \\
\end{array}$ \\
\hline
\end{tabular}

Tabla 2: Bandas principales en los espectros infrarrojo del Acrílico Gel Medium ALBA, pintura acrílica Alba con Blanco de Titanio (PW6) y el barniz acrílico W\&N.

\begin{tabular}{|c|c|c|c|}
\hline $\begin{array}{c}\text { Acrílico Gel Medium Alba } \\
\tilde{\mathbf{v}}\left(\mathrm{cm}^{-1}\right)\end{array}$ & $\begin{array}{c}\text { Pintura acrílica Alba con Blanco } \\
\text { de Titanio (PW6) } \\
\tilde{\mathbf{v}}\left(\mathrm{cm}^{-1}\right)\end{array}$ & $\begin{array}{c}\text { Barniz acrílico W\&N } \\
\tilde{\mathbf{v}}\left(\mathrm{cm}^{-1}\right)\end{array}$ & Asignación \\
\hline- & $3700-3000$ & - & Vibración de tensión del $\mathrm{O}-\mathrm{H}$ \\
\hline 2956 & 2953 & 2960 & $\begin{array}{l}\text { Vibración de tensión antisimétrica } \\
\text { del metilo }\end{array}$ \\
\hline 2934 (hombro) & 2934 (hombro) & 2935 (hombro) & $\begin{array}{l}\text { Vibración de tensión antisimétrica } \\
\text { del metileno }\end{array}$ \\
\hline 2874 & 2872 & 2875 & $\begin{array}{l}\text { Vibración de tensión simétrica del } \\
\text { metilo }\end{array}$ \\
\hline 1725 & 1726 & 1724 & Vibración de tensión $\mathrm{C}=\mathrm{O}$ en ésteres \\
\hline 1449 y 1436 & 1448 y 1437 & 1470 & $\begin{array}{l}\text { Vibración de flexión antisimétrica } \\
\text { C-H del metilo }\end{array}$ \\
\hline 1386 & 1385 & 1389 y 1368 & $\begin{array}{l}\text { Vibración de flexión simétrica } \mathrm{C}-\mathrm{H} \\
\text { del metilo }\end{array}$ \\
\hline 1235 & 1238 & 1237 & \multirow{6}{*}{$\begin{array}{l}\text { Vibraciones de tensión } \\
\text { C-O y C-C }\end{array}$} \\
\hline 1162 y 1143 & 1160 y 1147 & 1144 & \\
\hline 1065 & 1066 & 1062 & \\
\hline 989 & 992 & 993 & \\
\hline 962 & 961 & 967 & \\
\hline 942 & 942 & 946 & \\
\hline 843 & 842 & 879 & Vibración de torsión del $\mathrm{CH}_{2}$ \\
\hline 755 & - & 749 & $\begin{array}{l}\text { Vibración de flexión } \mathrm{C}-\mathrm{H} \text { fuera del } \\
\text { plano }\end{array}$ \\
\hline- & 483 & - & Vibración de tensión del Ti-O \\
\hline
\end{tabular}


Tabla 3: Bandas principales en los espectros infrarrojo de las muestras de pintura al óleo con Azul de Prusia (PB27).

\begin{tabular}{|c|c|c|c|}
\hline $\begin{array}{l}\text { Óleo Utrecht Oil Color } \\
\qquad \tilde{\mathbf{v}}\left(\mathrm{cm}^{-1}\right)\end{array}$ & $\begin{array}{c}\text { Óleo Louvre Oil } \\
\tilde{\mathbf{v}}\left(\mathrm{cm}^{-1}\right)\end{array}$ & $\begin{array}{c}\text { Óleo Winsor \& Newton Winton } \\
33 \\
\tilde{\mathbf{v}}\left(\mathrm{cm}^{-1}\right)\end{array}$ & Asignación \\
\hline $3500-3200$ & $3500-3200$ & $3500-3200$ & Pigmento \\
\hline 2921 & 2924 & 2923 & $\begin{array}{l}\text { Vibración de tensión antisimétrica del } \\
\text { metileno }\end{array}$ \\
\hline 2852 & 2854 & 2852 & $\begin{array}{l}\text { Vibración de tensión simétrica del } \\
\text { metileno }\end{array}$ \\
\hline 2083 & 2086 & 2078 & Pigmento \\
\hline 1739 & 1740 & 1736 & Vibración de tensión $\mathrm{C}=\mathrm{O}$ \\
\hline 1613 & 1607 & 1611 & Pigmento \\
\hline 1464 & - & - & Vibración de flexión del metileno \\
\hline- & 1413 & 1413 & $\mathrm{CaCO}_{3}$ (tiza) \\
\hline 1177 & - & - & $\mathrm{BaSO}_{4}$ \\
\hline 1069 & - & - & $\mathrm{BaSO}_{4}$ \\
\hline 983 & 976 & 978 & $\mathrm{BaSO}_{4} /$ Pigmento \\
\hline- & 871 & 878 & $\mathrm{CaCO}_{3}$ (tiza) \\
\hline- & 713 & 728 & $\mathrm{CaCO}_{3}$ (tiza) \\
\hline 637 & - & - & $\mathrm{BaSO}_{4}$ \\
\hline 606 & 604 & 603 & $\mathrm{BaSO}_{4} /$ Pigmento \\
\hline 495 & 498 & 495 & Pigmento \\
\hline
\end{tabular}

Recebido em: 27/02/2018

Aprovado em: 29/06/2018

Publicado em: 30/11/2018 